\title{
EFEITOS DO GRAU DE COZIMENTO NA QUALIDADE DE CORTES DE Supraspinatus ACONDICIONADO A VÁCUO EM EMBALAGEM COOK-IN
}

\author{
José Ricardo GONÇALVES ; ; Ana Lúcia da Silva Corrêa LEMOS
}

\begin{abstract}
RESUMO
Foi estudado o efeito do grau de cozimento na cor, perda de peso e força de cisalhamento em cortes no músculo Supraspinatus. Os músculos foram excisados de 18 meias-carcaças de Nelore e pesados antes e após retirada do excesso de tecido conjuntivo externo para cálculo de rendimento (porção comestivel). Em cada corte foram medidos o $\mathrm{pH}$ final e a cor, fazendo-se, individualmente, o acondicionamento em embalagens tipo cook-in. O cozimento foi feito em tacho com água observando-se as temperaturas internas finais (ponto frio) correspondentes ao cozimento "mal-passado", "ao ponto" e "bem-passado" e tempos de cozimento estimados, previamente, para promover a pasteurização (respectivamente, 60-62 C/300min, 70-72 C/120min. e 75-77 C/90min). Para cada tratamento foram destinados 6 músculos de pesos similares. Os dados obtidos indicam que a cor produto "mal-passado" foi ligeiramente mais vermelha que a do produto "ao ponto", mas o produto "bem-passado" foi fortemente afetado pelo tratamento. Como era esperado, o produto "bem-passado" apresentou maior perda de peso na cocção em relação aos produtos "ao ponto" e "mal-passado" (34,07, 24,83, e 21,66\%, respectivamente). Os valores da força de cisalhamento aumentaram do produto "malpassado" para o "bem-passado" (4,71,5,57, e 6,03kgf, respectivamente), sendo o "mal-passado" classificado como macio.
\end{abstract}

Palavras-chave: músculo bovino; embalagem cook in; grau de cozimento; alterações de cor; perdas na cocção; força de cisalhamento.

\section{SUMMARY}

EFFECTS OF COOKING DEGREE ON QUALITY OF VACUUM-PACKED Supraspinatus CUTS. The effects of the degree of cooking on color, cooking weight loss and shear force of beef supraspinatus muscle was evaluated. The muscles were extracted from eighteen Nellore carcasses sides without trimming. Each muscle was weighed before and after trimming to estimate the yield. The ultimate $\mathrm{pH}$ and color were achieved in the fresh cuts. The cuts were individually vacuum packed into cook-in bags and placed in a water bath in order to obtain the rare, medium and welldone final temperatures (coldest point) and pasteurized products. Each treatment was performed on groups of six muscles of similar weight. Data obtained indicated that color of rare product was slightly redder than medium product but welldone product was strongly affected by treatment. As expected, welldone product showed higher cooking loss than rare and medium products $(34.07,24.83$ and $21.66 \%$, respectively). Shear force values increased from rare to welldone cooking grade $(4.71,5.57$ and $6.03 \mathrm{kgf}$, respectively) being rare product classified as tender beef.

Keywords: beef muscle; cook in bag; rare, medium and welldone products; color; cooking loss; shear force.

\section{1- INTRODUÇÃO}

O grau de cozimento é definido por uma combinação de tempo e temperatura de aquecimento, cuja intensidade não só atua sobre a destruição de microrganismos e enzimas, mas também modifica as propriedades organolépticas e nutricionais do produto cozido [9].

Comumente, os processos industriais utilizam ar ou água como meio de aquecimento e diferentes temperaturas finais de cozimento, conforme o objetivo desejado. Há também um certo interesse da indústria em processos cujo consumo de energia é mínimo. Dentre os recentes desenvolvimentos, estão os processos que mantém uma diferença de temperatura entre o produto e o meio de aquecimento (delta T), comparado com aqueles convencionais, que fixam a temperatura do meio de aquecimento durante o tempo de duração do cozimento [2].

Recebido para publicação em 16/04/2004. Aceito para publicação em 04/05/2005(001326).

Centro de Tecnologia de Carnes, Instituto de Tecnologia de Alimentos. Campinas-SP.E-mail:jricardo@ital.sp.gov.br

A quem a correspondência deve ser enviada.
Para a carne in natura, a cor é o atributo mais observado na hora da compra, o odor é destacável durante o cozimento e a textura é mais importante no ato da mastigação da carne cozida. Porém, no aspecto de qualidade degustativa, a retenção de suco contribui para um produto cozido suculento, transmitindo uma sensação de prazer ao consumidor [12, 19]. Este, em geral, baseia-se numa classificação popular para preparar o seu produto no lar ou para consumi-lo num restaurante ou estabelecimento comercial do gênero: "mal-passada"; "ao ponto" ou "bem-passada" .

A literatura define a carne bovina cozida a temperatura interna de $60^{\circ} \mathrm{C}$ como sendo "mal-passada" ; a $71^{\circ} \mathrm{C}$, "ao ponto" ; e a 77 C, "bem-passada" [12, 18].

Há vários trabalhos científicos sobre os efeitos da cocção na qualidade da carne bovina, notadamente com músculos potencialmente macios e de maior valor comercial, como os do traseiro. Isso é menos freqüente dentre os músculos do dianteiro, embora alguns apresentem maciez intermediária, como o Supraspinatus e o Triceps brachii [10]. São músculos com maior teor de colágeno e que requerem um método de cozimento apropriado para destacar as suas qualidades. O baixo grau de marmoreio favorece a aparência do corte cozido [2]. 
No Brasil, os cortes de dianteiro são chamados popularmente de "carne de segunda", geralmente comercializados in natura. São cortes de rendimento inferior e menor valor comercial, mas com boas propriedades nutricionais. Diante da eventual redução de oferta das carnes nobres por causa da demanda crescente das exportações é preciso encontrar soluções para agregar valor à "carne de segunda" e viabilizar o seu consumo no mercado interno [7].

O aproveitamento de tais cortes embalados a vácuo, cozidos e estocados sob refrigeração poderia ampliar o atendimento às necessidades de alimentação em restaurantes comerciais e indústriais, hotéis, escolas, hospitais e outros estabelecimentos institucionais, nos quais a carne bovina representa $60 \%$ do total de produtos cárneos consumidos [1].

Em nota prévia, GONÇALVES, OLIVEIRA \& BARBIERO [6] apresentaram resultados sobre o cozimento "mal-passado" e "bem-passado" do Supraspinatus acondicionado em embalagem cook-in, utilizando um tacho com água como meio de aquecimento. Os autores também estimaram o tempo de cozimento necessário em cada tratamento para a obtenção de um produto pasteurizado, seguro para consumo. Neste particular, o sistema cook-in pode dar uma boa contribuição, evitando o manuseio pós-processamento. Por se tratar de uma tecnologia simplificada existe a expectativa de poder utilizá-la em pequenos abatedouros e indústrias de processamento.

Nesta proposta pretende-se avaliar a qualidade do mesmo corte pré-acondicionado a vácuo, em embalagem cook-in, quando submetido as seguintes condições de cozimento: "mal-passado", "ao ponto" e "bem-passado".

\section{2- MATERIAL E MÉTODOS}

Os músculos foram retirados de 18 meias-carcaças de animais Nelore (30-36 meses), submetidos ao mesmo manejo de alimentação e sanidade, abatidos no CTC em duas datas distintas e com o uso de estimulação elétrica de baixa voltagem, imediatamente após a sangria (80v/90s). O resfriamento das carcaças foi feito de forma convencional, seguido de desossa $24 \mathrm{~h}$ pós-morte, sendo os músculos pesados antes e depois da retirada do excesso de tecido conjuntivo externo para o cálculo de rendimento em corte limpo (porção comestivel). Na seqüência cada corte foi submetido as determinações de $\mathrm{pH}_{2, \ldots}$ e cor ( $\mathrm{L}^{*}, \mathrm{a}^{*} \mathrm{e} \mathrm{b}^{*}$, respectivamente para luminosidade e intensidades de vermelho e amarelo), conforme especificações da CIE [13]. Os cortes foram acondicionados a vácuo em embalagens individuais do tipo cook-in (Cryovac $\left.{ }^{\circledR}\right)$ e separados, aleatoriamente, em grupos de 3 unidades por tratamento. Posteriormente, foram cozidos em tacho com água sob condições distintas, observando as temperaturas internas finais correspondentes ao cozimento "mal-passado", "ao ponto" e "bem-passado" e tempos de cozimento estimados por GONÇALVES, OLIVEIRA \& BARBIERO [6] para promover a pasteurização, isto é, 60$62 \mathrm{C} / 300 \mathrm{~min}, 70-72 \mathrm{C} / 120 \mathrm{~min}$ e $75-77 \mathrm{C} / 90 \mathrm{~min}$., res- pectivamente (cada tratamento foi replicado uma segunda vez).

Para monitorar a temperatura, termopares tipo $\mathrm{T}$ foram imersos na água e, no caso dos cortes, inseridos no ponto frio através de um sistema auto-vedante instalado na extremidade do fundo das embalagens, o qual permitiu a manutenção das condições de vácuo após o fechamento. Após o cozimento foi feito o resfriamento em água corrente (23-25 C) até o produto atingir 25-27 C no ponto frio, sendo posteriormente encaminhado para as determinações de perda de peso na cocção, cor e força de cisalhamento.

A descrição dos métodos está apresentada abaixo:

- $\quad$ pH: Eletrodo de punção acoplado em potenciômetro devidamente calibrado, marca DigimedDM 2, obtendo-se o valor médio de cada corte a partir de 3 leituras.

- Perda de Peso na Cocção: medida em função da diferença de peso do corte limpo (antes e após o cozimento), expressando-se o resultado em porcentagem.

- Cor: Espectrofotômetro Minolta, modelo Cm 508-d (iluminante $\mathrm{D}_{c}$, e 10 ), obtendo-se o valor médio de cada corte a partir de 5 leituras feitas na superficie externa do corte in natura e, após o cozimento, nos respectivos bifes de $2,5 \mathrm{~cm}$ de espessura.

Força de cisalhamento: Texturômetro TA-XT 2i, devidamente calibrado com peso padrão (rastreável), operando com lamina Warner-Bratzler, num ambiente a $20-25^{\circ} \mathrm{C}$. De cada corte cozido foram retiradas 12 amostras cilindricas de $2,5 \mathrm{~cm}$ de comprimento por $1,27 \mathrm{~cm}$ de diâmetro, sendo obtido o valor médio (em kgf) para a medida perpendicular as fibras.

Os resultados foram submetidos à análise de variância (ANOVA) e a diferença significativa entre as médias determinada pelo teste LSD ("Least Significant Difference") ao nivel de 5\%, utilizando-se o software Statistic 5.0.

\section{3- RESULTADOS E DISCUSSÃO}

O Supraspinatus apresentou um valor médio do $\mathrm{pH}_{3 .}=5,62 \pm 0,03$, comum na carne bovina in natura, cuja faixa considerada normal é 5,4-5,7 [12]. O peso bruto médio de $1475,8 \pm 74,4 \mathrm{~g}$, foi superior em $8,6 \%$ ao valor de $1358 \mathrm{~g}$ publicado por GONÇALVES, OLIVEIRA \& BARBIERO [6] para animais Nelore criados em igual sistema de produção e abatidos e desossados nas mesmas condições deste experimento. O resultado também está $7,4 \%$ acima de $1374 \mathrm{~g}$, valor obtido por BUTTERFIELD \& MAY [3] para o mesmo tipo de músculo (possivelmente, em outras condições experimentais). Essas diferenças parecem aceitáveis, mesmo em se tratando de animais de mesma espécie, raça e sexo, pois a variação do peso do músculo depende ainda do peso vivo ao abate e de outras variáveis. Por outro lado, o rendimento de $68 \pm 3,3 \%$ em 
corte limpo, embora razoável, foi inferior aos $74,6 \%$ apresentado por GONÇALVES, OLIVEIRA \& BARBIERO [6] para o mesmo peso final de 1000 g. No aspecto econômico essa diferença para menos significa algo em torno de $97 \mathrm{~kg}$ para cada tonelada produzida do respectivo corte, apenas considerando a operação de limpeza. A definição do melhor rendimento para o corte limpo vai depender da padronização do grau de limpeza em função da qualidade desejada, isto é, da maior ou menor retirada de tecido conjuntivo externo. Os valores médios \pm erro padrão de $\mathrm{L}^{*}, \mathrm{a}^{*} \mathrm{e} \mathrm{b}$ obtidos para o corte in natura foram, respectivamente, $31,55 \pm 1,46 ; 15,46 \pm 1,19$; e $3,33 \pm 1,19$ e refletem as características da carne bovina em termos de cor, embora alguma variação possa existir por fatores diver$\operatorname{sos}[17]$.

A Tabela 1 mostra a variação dos parâmetros de cor após o cozimento do corte.

TABELA 1 - Valores médios \pm erro padrão de $L^{*}$, a* e b*, após o cozimento dos cortes nos três tratamentos em estudo (1)

\begin{tabular}{|c|c|c|c|}
\hline Tratamento & $\mathrm{L}^{*}$ & $a^{*}$ & $b^{*}$ \\
\hline $\begin{array}{l}\text { "Mal-passado" } \\
\text { (60-62"C/300min) }\end{array}$ & $54,55 \pm 0,66^{a}$ & $13,28 \pm 0,41^{a}$ & $16,55 \pm 0,55^{a}$ \\
\hline $\begin{array}{c}\text { "Ao Ponto" } \\
\text { (70-72 } 2 \text { C/120min) }\end{array}$ & $51,84 \pm 0,75^{b}$ & $12,47 \pm 0,42^{\mathrm{a}}$ & $17,20 \pm 0,86^{a}$ \\
\hline $\begin{array}{l}\text { "Bem-passado" } \\
\left(75-77^{\circ} \mathrm{C} / 90 \mathrm{~min}\right)\end{array}$ & $51,23 \pm .0,41^{\mathrm{b}}$ & $7,78 \pm 0,17^{b}$ & $14,17 \pm 0,93^{b}$ \\
\hline
\end{tabular}

Entre os três tratamentos estudados observou-se que as variações de $\mathrm{L}^{*} \mathrm{e} \mathrm{b}^{*}$ foram moderadas. A mudança mais substancial foi a redução de $41,4 \%$ do valor de $a^{*}$, do cozimento "mal-passado" para o "bem-passado", mostrando uma brusca transformação do rosa avermelhado para o marron acinzentado. O tratamento "ao ponto" apresentou uma coloração rosada, mesclada com algumas áreas mais escuras, caracterizando a desnaturação parcial da mioglobina (estatisticamente, não houve diferença significativa, comparado ao tratamento "malpassado"). Esta tendência também foi observada por VAUDAGNA et al. [20], utilizando o método Hunter. Os autores concluíram que os valores de $L \mathrm{e} b$ não foram afetados $(\mathrm{p}<0,05)$ no cozimento do Semitendinosus com temperaturas entre 50 e $65 \mathrm{C}$, mas a redução do valor de a foi drástica (isto é, $44 \%$ ) com o aumento de temperatura, no referido intervalo.

Se a comparação desses parâmetros for feita a partir do corte in natura, percebe-se que $\mathrm{L}^{*} \mathrm{e} \mathrm{b}^{*}$ foram drasticamente aumentados após o cozimento, enquanto o valor de $\mathrm{a}^{*}$ sofreu reduções crescentes à medida que o grau de cozimento foi intensificado. Isso é conseqüência da transformação do pigmento vermelho para marronacinzentado. Uma forma de avaliar a evolução das perdas da cor vermelha antes e depois do cozimento é comparar os valores de $\mathrm{a}^{*}$ em cada tratamento com os da carne in natura e expressá-los em percentagem (Figura 1). De fato, o tratamento "bem-passado" foi capaz de reduzir o valor de a* em mais de $50 \%$. Os outros dois tratamentos sofreram reduções mais moderadas e não apresentaram diferenças significativas entre si $(p<0,05)$, embora alguma transição tivesse sido observada no tratamento "ao ponto" . A explicação para o caso é que a desnaturação da mioglobina começa a ser intensificada a partir de $70 \mathrm{C}$ e está praticamente concluída na temperatura final do tratamento bem-passado [19].

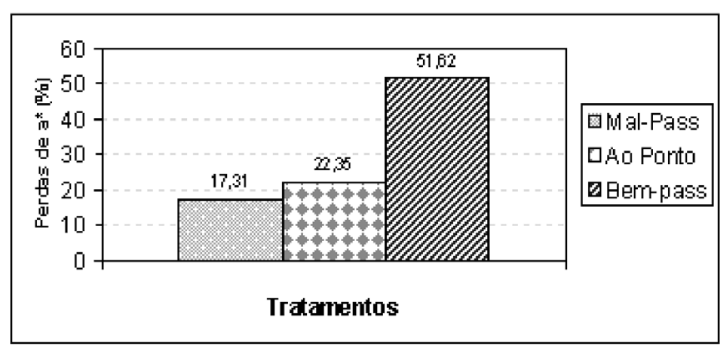

FIGURA 1 - Perdas percentuais do valor de a* para cada tratamento em comparação com o corte in natura

Em termos de cor, o cozimento mal-passado oferece maior flexibilidade na preparação do produto para consumo. Para os que desejam o produto tipicamente malpassado basta reaquecê-lo em forno de microondas antes de servir. Àqueles que têm preferência pela cor marrom na superficie do produto o reaquecimento pode ser feito em chapa a 150-180 C, condição que favorece a ocorrência das reações de caramelização e de Maillard [5].

A Tabela 2 apresenta alguns resultados interessantes sob outros aspectos de qualidade da carne. O grau de cozimento "bem-passado", quando analisado isoladamente, proporcionou uma perda de peso elevada, embora não tenha atingido o valor que ocorre normalmente em cozimentos, que é cerca de $40 \%$ [11].Em termos relativos esta perda foi superior em $57 \%$ e $37 \%$, respectivamente, às do cozimento "mal-passado" e "ao ponto". Tais diferenças são consideráveis e, provavelmente, não deixariam de ser detectadas numa avaliação sensorial de suculência, mesmo ao nível de consumidor. Porém, a sensação de perda de suculência pode ser minimizada nos tratamentos mais severos, utilizando-se o suco exsudado durante o cozimento (e retido na embalagem ) como base na rápida preparação de molhos para a complementação do prato.

Segundo relato de LEDWARD [12], o tratamento do Pectoralis profundus durante $1 \mathrm{~h}$ entre 30 e $80 \mathrm{C}$ mostrou que as perdas na cocção aumentaram quase linearmente com a temperatura, particularmente entre 50 e $80 \mathrm{C}$. Nas temperaturas de 60 e $80 \mathrm{C}$, as perdas ficaram em cerca de $22 \%$ e $38 \%$, respectivamente. O referido músculo foi retirado de animais jovens (24-36 meses) e apresentava $\mathrm{pH}$ normal, como no presente experimento. Mais recentemente, VAUDAGNA et al. [20], trabalhando com novilhos (provavelmente de origem européia), estudaram o cozimento do Semitendinosus em água, acondicionado a vá- 
cuo em embalagem cook in, numa faixa entre 50 e $65 \mathrm{C}$, combinada com tempos de 90 a 390min. Nas condições específicas de $60 \mathrm{C} / 270 \mathrm{~min}$, as perdas na cocção aproximaram-se de $17 \%$. Quando a temperatura foi aumentada para $65 \mathrm{C}$ e o tempo reduzido para $90 \mathrm{~min}$, tais perdas atingiram o valor máximo dentre todos os tratamentos, isto é, $20 \%$. Os autores concluíram que, as perdas na cocção crescem com a temperatura, especialmente quando ela supera $60 \mathrm{C}$, não dependendo significativamente do tempo de cozimento. $\mathrm{O}$ fenômeno pode ser atribuído ao aumento da desnaturação do colágeno e da actina, com encolhimento longitudinal das fibras e diminuição no comprimento do sarcômero [15]. Embora a comparação seja feita com tipos diferentes de músculo, os dados da Tabela 2 acompanham a tendência identificada pelos autores.

TABELA 2 - Valores médios de atributos de qualidade dos Supraspinatus após o cozimento "mal-passado", "ao ponto" e bem-passado" (1)

\begin{tabular}{lcc}
\hline Tratamentos & Perdas na cocção (\%) & Cisalhamento (Kgf) \\
\hline $\begin{array}{c}\text { "Mal-passado" } \\
\left(60-62^{\circ} \mathrm{C} / 300 \mathrm{~min}\right)\end{array}$ & $21,66 \pm 0,78^{\mathrm{a}}$ & $4,71 \pm 0,16^{\mathrm{a}}$ \\
& & \\
"Ao Ponto" & $24,83 \pm 0,61^{\mathrm{b}}$ & $5,57 \pm 0,07^{\mathrm{b}}$ \\
$\left(70-72^{\circ} \mathrm{C} / 120 \mathrm{~min}\right)$ & & $6,03 \pm 0,19^{\mathrm{b}}$ \\
"Bem-passado" & $34,07 \pm 1,09^{\mathrm{c}}$ & \\
$\left(75-77^{\circ} \mathrm{C} / 90 \mathrm{~min}\right)$ & \\
$\begin{array}{l}\text { (1) Médias com letras diferentes na mesma coluna são significativamente diferentes } \\
\text { (p<0,05). }\end{array}$
\end{tabular}

Ainda na Tabela 2, nota-se que a força de cisalhamento foi maior para o tratamento "bem-passado" e superior em $28 \%$ a do tratamento "mal-passado". Já no tratamento "ao ponto", a força de cisalhamento resultou em um valor intermediário, embora, estatisticamente, não tenha acusado diferença significativa $(\mathrm{p}<0,05)$ em comparação com a do tratamento "bem-passado". Ao adotar como referência o valor de 5,4kgf (Warner-Bratzler em amostras de $1,27 \mathrm{~cm}$ de diâmetro), estabelecido por CARPENTER [4] como limite para considerar a carne macia, apenas o tratamento "mal-passado" seria classificado como tal.

Uma outra observação é que a força de cisalhamento mostrou uma relação crescente e quase linear entre os tratamentos (Figura 2). Em princípio, há uma hipótese aceitável para explicar a ausência de uma linearidade verdadeira para o intervalo de temperatura utilizado no estudo (60-75 C). Conforme VARNAN \& SUTHERLAND [19], o aumento da força de cisalhamento com a temperatura ocorre em duas faixas distintas: 45-50 C e 65-70 C. Na primeira faixa o fenômeno é atribuído à desnaturação de proteínas miofibrilares, enquanto na segunda, ao encolhimento do colágeno perimisial. Nas temperaturas intermediárias a carne não chega a sofrer os efeitos da tensão nas fibras de colágeno. Por conseguinte, isso sugere uma certa descontinuidade no aumento da força de cisalhamento com a temperatura, descartando uma relação perfeitamente linear no intervalo 60-75 C.

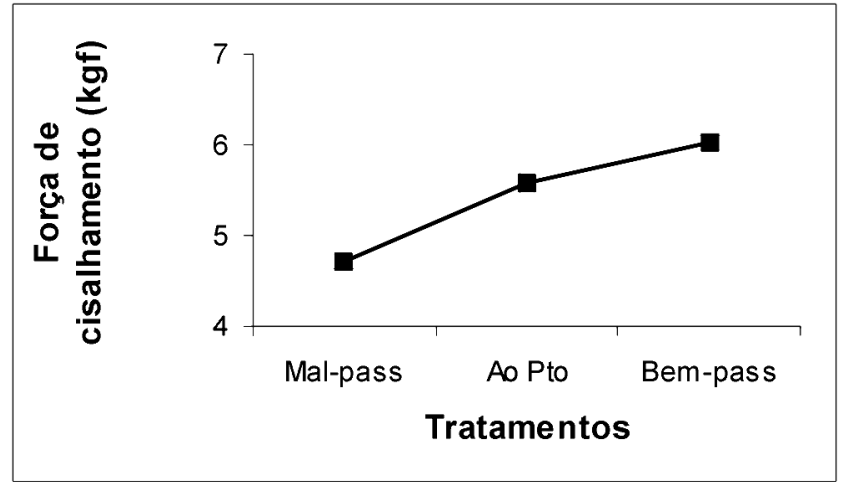

FIGURA 2 - Variação da força de cisalhamento em função dos tratamentos

PINTO NETO, BERAQUET \& CARDOSO [16], estudaram a maciez do filé-mignon (Psoas major) retirado dos mesmos animais e sob iguais condições de abate e resfriamento de carcaça utilizadas no presente estudo. As amostras foram cozidas em chapa aquecida a $150 \mathrm{C}$, até que a temperatura interna atingisse $74 \mathrm{C}$. A força de cisalhamento obtida 2 dias pós-abate foi medida no mesmo texturômetro e resultou no valor de 4,32kgf. Embora os autores tenham utilizado um outro meio de aquecimento e temperatura interna maior que a do tratamento "malpassado", a força de cisalhamento do filé-mignon foi muito parecida com a do tratamento "mal-passado" do supraspinatus $(4,32 \mathrm{kgf}$ versus $4,71 \mathrm{kgf})$. Isso reforça a recomendação geral de que o método por calor seco é mais apropriado para cortes potencialmente macios, enquanto que o método por calor úmido se destina a cortes com maior teor de colágeno [14]. Conforme HENRICKSON [8], o tempo e a temperatura são importantes, tanto para o enrijecimento da fibra muscular como para o amaciamento do colágeno. Porém, o fator temperatura é mais importante para o primeiro, enquanto o fator tempo é mais importante para o segundo caso.

\section{4 - CONCLUSÕES}

O Supraspinatus apresentou um bom rendimento em corte limpo, o qual poderá variar em função do peso bruto e da definição do grau de limpeza desejado. O grau de cozimento teve um efeito significativo nos atributos de qualidade, especialmente na perda de peso por cocção, que está associada à suculência do produto final. O cozimento com longo tempo e baixa temperatura ("malpassado") foi capaz de proporcionar a obtenção de um produto com textura macia, menor perda de líquido na cocção e menor redução da intensidade do vermelho, em comparação aos outros dois tratamentos.

É um corte com potencial para aproveitamento no mercado institucional na forma cozida (pasteurizada) e refrigerada, aproveitando as vantagens do sistema cookin, o qual restringe a manipulação do produto ao momento da preparação para consumo. O suco exsudado 
durante o cozimento e retido na embalagem pode ser utilizado como base na rápida preparação de molhos para a complementação do prato, minimizando a sensação de perda de suculência no tratamentos mais severos. Estas condições agregam valor e conveniência ao corte cozido por meio de uma tecnologia simplificada e que poderia ser utilizada em pequenos abatedouros ou indústrias de processamento.

À título de continuidade, sugere-se avaliar a vida útil do produto com base em análise sensorial e algumas determinações físico-químicas.

\section{5 - REFERÊNCIAS BIBLIOGRÁFICAS}

[1] BLISKA, F. M. M.; RAZOOK, A. G.; PITUCO, E. M.; ALLEONI, G. F.; COUTINHO, J. L. V. ; GONçALVES, J. R.; LEME, P. R. Prospecção de Demandas Tecnológicas na Cadeia Produtiva de Carne Bovina no Estado de São Paulo. Boletim Técnico n. 42, Instituto de Zootecnia. Nova Odessa, São Paulo.1998, 73p.

[2] BOLES; J. A.;SWAN, J. E. Heating method and final temperature affect processing characteristics of beef semimembranosus muscle. Meat Science, v. 62, p. 107$112,2002$.

[3] BUTTERFIELD, R.M.; MAY, N.D.S. Muscles of the Ox. 1 ed. University of Queensland Press. Queensland. Australia. 1966, 164p.

[4] CAPENTER, J. W. A comparison of slaught, carcass, palatability and meat processing characteristics of mater buffalo and Charolais bulls. Water Buffalo Newsletter, v. 2, p.4-6, Florida. USA., 1988.

[5] FOX, J. B. Los pigmentos de la carne. In: Price, J. F.; SCHWEIGERT, B. S. Ciência de la Carne y de Los Productos Cárnicos. Zaragoza. España: Editorial Acribia,1994, p.175-198

[6] GONÇALVES, J.R.; OLIVEIRA, J.; BARBIERO, R. Características da carne (músculo supraspinatus) embalada a vácuo e cozida até a condição de "malpassada" e "bem-passada". Campinas. Braz. J. Food Technol., v. 5, p. 67-71,2002.

[7] GONÇALVES, J.R.; ALMEIDA, G.S. Brazilian Beef Exports and its Main Markets: a brief analysis. FLEISCHWIRTSCHAFT INTERNATIONAL. Frankfurt, Germany. n.4, November, 2003.

[8] HENRICKSON, R. L. Meat, Poultry and Seafood Technology. Prentice-Hall, Inc. Englewood Cliffs, New Jersey, Chapter 17, p.215-227, 1978.
[9] HOLDSWORTH, S. D. Thermal processing of packaged foods. 1 " edition. Blackie Academic and Professional. London. UK. Chapter 3, p.70-97, 1997.

[10] JUDGE, M.D.; ABERLE, E.D; FORREST, J.C.; HEDRICK, H.B.; MERKEL, R.A. Principles of meat science. 2ed. USA: Kendall/Hunt Publishing Company, 1989.

[11] LAWRIE, R. Developments in Meat Science-4. Elsevier Science Publishers Ltd. Great Britain, Chapter 3, p.63$171,1988$.

[12] LEDWARD, D.A. Meat. In: PRIESTLEY, R.J. (Ed.) Effects of Heating on Foodstuffs. Applied Science Publishers. England, Chapter 5; p.121-157, 1979.

[13] MACDOUGALL, D.B. Color of meat. In: PEARSON, A.M., DUTSON, T.R. Advances in meat research Quality attributes and their measurement in meat, poultry and fish products. Mariland: Aspen Publishers, Inc., Cap. 3, v.9, p. 79-92, 1999.

[14] Pearson, A.M; TAUber, F.V. Processed Meats. 2 ed. AVI Publising Co. Inc., Westport, Connecticut. USA, Cap. 5; p.121-157, 1984.

[15] OFFER, G., RESTALL, D. , TRINICK, J. Water-holding in meat. In: BAILEY, A J.(Ed.). Recent advances in chemistry of meat. London, U.K. The Royal Society of Chemistry, p..45-94, 1984

[16] PINTO NETO, M.; BERAQUET, N. J.; CARDOSO, S. Effects of electrical stimulation on muscle psoas major from Bos Indicus carcasses suspended by the aitch bone or chilled very fast after hot boning. In: $49^{\text {"n }}$ INTERNATIONAL CONGRESS OF MEAT SCIENCE AND TECHNOLOGY.2003, August 31-06 September. Campinas, Brazil . Proceedings. Vol. I-p.163-164.

[17] RENERRE, M. Review: factors involved in the discoloration of beef meat. Internacional Journal of Food Science and Technology, v. 25, p.613-630,1990.

[18] SAVEL, J. W.; SMITH, G.C. Laboratory Manual for Meat Science. Sixt Edition. 226p. American Press. USA.1998.

[19] VARNAN, A.H.; SUTHERLAND, P.J. Meat and meat products. Technology, chemistry and microbiology. Chapman \& Hall. London. 1 ed.,Vol 3, Food Products Series, 430p., 1995.

[20] VAUDAGNA, S. R.; SÁNCHEZ, G; NEIRA, M. S.; INSANI, E. M.; PICALLO, A. B.; GALLINGER, M. M.; LASTA, J.A. Sous vide cooked beef muscles: effects of low temperature-long time (LT-LT) treatments on their characteristics and storage stability. International Journal of Food Science and Technology, v. 37, p.1$17,2001$. 